\title{
Effect of glabridin on collagen deposition in liver and amelioration of hepatocyte destruction in diabetes rats
}

\author{
MANARAS KOMOLKRIENGKRAI ${ }^{1}$, JONGDEE NOPPARAT ${ }^{1}$, URAPORN VONGVATCHARANON ${ }^{1}$ \\ VIPAVEE ANUPUNPISIT $^{2}$ and WIPAPAN KHIMMAKTONG ${ }^{1}$ \\ ${ }^{1}$ Department of Anatomy, Faculty of Science, Prince of Songkla University, Hatyai, Songkhla 90110; \\ ${ }^{2}$ Department of Anatomy, Faculty of Medicine, Srinakharinwirot University, Bangkok 10110, Thailand
}

Received April 10, 2018; Accepted October 12, 2018

DOI: $10.3892 /$ etm.2019.7664

\begin{abstract}
Abnormalities in insulin hormone levels leads to a hyperglycemic condition of diabetic mellitus. Hyperglycemia seriously induces organ and system destructions. The excessive accumulation of collagen fiber deposits occurs in inflammatory and reorganization processes of chronic liver diseases in type I insulin-dependent diabetes. Regarding the research objective, glabridin (GLB), an active compound of licorice, was used as a daily supplement $(40 \mathrm{mg} / \mathrm{kg})$ in order to decrease hepatocyte destruction and collagen deposition in liver tissue of diabetic animals induced by streptozotocin. A total of 40 were randomly allocated to five groups (each, $\mathrm{n}=10$ ), control, control treated with GLB (GLB), diabetic rats (DM) injected with single dose of streptozotocin $(60 \mathrm{mg} / \mathrm{kg})$ to induce a diabetic condition, diabetic rats receiving GLB (DM+GLB; $40 \mathrm{mg} / \mathrm{kg}$ ) and diabetic rats treated with glibenclamide (DM+GL; $4 \mathrm{mg} / \mathrm{kg}$ ). Characteristic histopathological changes in liver cells and tissues of rats were determined by Masson's trichrome staining and transmission electron microscopy (TEM). Western blotting was used to detect the expression of the key markers, collagen type I and fibronectin proteins. The histological investigation of liver tissue of the DM group revealed that the collagen fiber deposition was increased in the periportal, pericentral and perisinusoidal spaces compared with controls. Hepatocytes appeared as small and fragmented cells in TEM examination. Collagenization of the perisinusoidal space was recently demonstrated to represent a new aspect of the microvascular abnormalities and liver fibrosis. Healthy hepatocytes with round nucleus were observed following supplementation of glabridin. In addition, collagen fiber deposition was reduced in the area adjacent to the perisinusoidal space. The expression of collagen type I and
\end{abstract}

Correspondence to: Dr Wipapan Khimmaktong, Department of Anatomy, Faculty of Science, Prince of Songkla University, Hatyai, Songkhla 90110, Thailand

E-mail: wipapan.k@psu.ac.th

Key words: diabetes mellitus, streptozotocin, glabridin, licorice, collagen, fibrosis, transmission electron microscope fibronectin decreased strongly following glabridin supplementation in DM+GLB rats compared with DM rats, indicating that the hepatic tissue reorganization regained its normal morphology. These findings suggest that it may be beneficial to examine the role of glabridin as a therapeutic agent in diabetes treatment in future research.

\section{Introduction}

Extracellular matrix (ECM) accumulation or changing of the condition of the extracellular matrix is a common pathological reaction to tissue abuse acting as hyperglycemia (1). ECM is dissimilar quantitatively and qualitatively within various tissue and organs. The changes of which lead to diabetes-induced organ and system failure, resulting in nephropathy, retinopathy, and diabetic cardiomyopathy (2). It comprises protein fibers such as collagens and elastins implanted in an amorphous compound of proteoglycan molecules. Chronic hyperglycemia can cause disturbances of morphology and biochemistry of ECM that are associated with a lost function in target organs and induces the increase of reactive oxidative species (ROS) production (3). Furthermore, the excessive accumulation of fat and oxidative stress can influence diabetic pathology and complications, including diabetic liver damage (4). Oxidative stress occurs when the production of ROS exceeds its clearance. It constitutes a characteristic feature of hepatic injuries (5). At this time, the hepatocytes are damaged and Kupffer cells are activated. The inflammatory cells and platelets release cytokines and growth factors that result in fibrogenesis $(6,7)$. Fibrosis is the deposit of connective tissue by the liver in response to injury commonly found in most chronic inflammatory liver diseases.

Liver fibrosis entails substantial alterations in both composition and amount of the deposited ECM (4). This process comprises an inflammatory response and limits ECM deposition. The inflammatory cytokines and growth factors induce hepatic stellate cell (HSC) activation and cellular production such as transforming growth factor (TGF)- $\beta$ (8), collagen (type I, III, and IV) (9), and fibronectin $(10,11)$. TGF- $\beta$ creates an accumulation of pro-fibrotic factors at the site of injury, which is common in several human fibrotic states (12). Collagens type I and IV are associated with the healing process of diabetic liver tissue (6). Therefore, the appearance and detection of 
collagen deposition in liver tissues might be the key markers for diagnosis $(13,14)$. Fibronectin participates in the progression of the fibrotic state and supports other matrix proteins correlating with cell cycle development. Furthermore, it also cooperates in cell proliferation and adhesion (15).

The deposition of collagen is the initiating event triggering liver fibrosis $(16,17)$. Concerning acute hepatic injury, HSCs (HSCs) differentiate from pericytes into myofibroblasts for reconstruction of the ECM. Conversely, in chronic hepatic injury, HSCs occupy the space of Disse to serve the progression of excessive ECM production and decrease ECM metabolism (11). Movement and aggregation of HSCs at the area of tissue reconstruction activate ECM deposition and alter its degeneration (9). If the hepatic injury continues, the liver regeneration eventually fails, and abundant ECM fills the place occupied by hepatocytes leading to collagenization of liver tissue (16).

Licorice has been reported to protect against hepatic injury (18). It also prevents oncogenesis caused by abnormal hormones or toxic substances and ameliorates free radical-induced oxidative of kidney damage (19). Glabridin ishe main active component of licorice (Glycyrrhiza glabra). It is a polyphenolic flavonoid that displays estrogenic, antimicrobial, anti-fatigue and anti-proliferative activity activities in human breast cancer cells (20). In addition, it reduces inflammation and alters melanogenesis (21). Glabridin displays potent antioxidative and superoxide-scavenging activities in biological membranes (22). It can prevent mitochondrial lipid peroxidation and protect respiratory enzyme activities against oxidative stresses in the mitochondrial electron transport system (22). Accumulating lines of evidence demonstrated that licorice has anti-inflammatory, anticancer, antioxidant and antimicrobial effects $(20,23)$. In particular, Jung et al (24) have previously evaluated the hepatoprotective effects of licorice extract and suggested that it could reduce liver injury by enhancing antioxidant and anti-inflammatory capacity in alcohol induced fatty liver disease. In addition, Wu et al (25), reported that the hypoglycemic effect of glabridin increased body weight, glucose tolerance and superoxide dismutase (SOD) activities in the liver, kidney and pancreas, whereas decreasing fasting blood sugar levels and malondialdehyde (MDA) content in the liver, kidney and pancreas in the STZ induced diabetic mice for 28 days. The morphological changes of liver cells and tissues that may result from the treatment and supplementation with glabridin remain to be elucidated. The present study aimed to evaluate the efficiency of glabridin on restoration and improvement of diabetic liver tissue on histological, ultrastructural changes and to determine the collagen type I and fibronectin protein expressions in streptozotocin (STZ)-induced diabetic rats. The results indicated that glabridin from licorice may affect the collagen deposition and the ECM accumulation and reverse the patterns of area-based liver tissue reorganization. Therefore, glabridin may have potential to repair the damaged diabetic liver.

\section{Materials and methods}

Induction and assessment of diabetes. The present study used 8-week-old male Wistar rats (weight, 200-250 g) provided by the Southern Laboratory Animal Facility, Prince of Songkla
University (Hatyai, Thailand). A total of 40 rats were housed in a controlled animal laboratory environment and maintained under a humidity of $(50 \pm 10 \%)$ in a 12 -h light/dark cycle $\left(25 \pm 2^{\circ} \mathrm{C}\right)$, with ad libitum access to standard rat chow and water. The experimental protocol used was approved by the Animal Ethics Committee of the Prince of Songkla University. Experimental diabetic rats were induced by single dose intraperitoneal injection of STZ (60 mg/kg; Sigma-Aldrich; Merck KGaA, Darmstadt, Germany) dissolved in $0.1 \mathrm{~mol} / 1$ citrate buffer. Control rats were injected with citrate buffer alone. Blood sugar level was measured and analyzed by one-touch glucometer (Accu-Check Active ${ }^{\circledR}$; Roche Diagnostics GmbH, Mannheim, Germany). Rats with blood sugar level > $250 \mathrm{mg} / \mathrm{dl}$ were used as the diabetic group. In order to monitor blood glucose levels, blood glucose was tested every week for 8 weeks. Control and diabetic rats were randomly divided into five groups (each, $n=10$; Fig. 1): Control rats receiving a balanced standard diet; glabridin control rats receiving the same diet supplemented with glabridin (GLB; purified $>98 \%$ by high-performance liquid chromatography analysis; Shaanxi Langrun Biotechnology Co., Ltd., Xi'an, China) in $0.5 \mathrm{ml}$ of $0.5 \%$ Tween 80 solution; diabetic rats receiving a balanced standard diet (DM); diabetic rats receiving a balanced standard diet supplemented with glabridin (DM+GLB) in $0.5 \mathrm{ml}$ of $0.5 \%$ Tween 80 solution; and diabetic rats treated with glibenclamide (Sigma-Aldrich; Merck KGaA; DM+GL) $4 \mathrm{mg} / \mathrm{kg}$ in $0.5 \mathrm{ml}$ of $0.5 \%$ Tween 80 solution in order to demonstrate the effectiveness of glabridin. All animals were clinically observed and weighed on a weekly basis. Following 8 weeks of glabridin supplementation, the animals were sacrificed and blood was drawn from the heart into sample tubes for screening of liver function. The liver function test was analyzed by the Southern Lab Center Saha Clinic (Songkhla, Thailand; using a Siemens ADVIA 1800 System Analyzer; Siemens Healthineers, Erlangen, Germany). Livers were removed, dissected, and immediately fixed in $10 \%$ formalin at room temperature for $24 \mathrm{~h}$ as a preparation for histological experiments.

Masson's trichrome staining. Following fixing the hepatic tissue samples in $10 \%$ formalin, they were dehydrated in graded series of ethanol through 70,80, 90, 95 and $100 \%$ with two changes for $1 \mathrm{~h}$ each. Three changes of xylene as clearing reagent for $30 \mathrm{~min}$ each were performed. Hepatic tissues were then embedded in paraffin, sliced into $5-\mu \mathrm{m}$ sections, and stained with Masson's trichrome at room temperature for $2 \mathrm{~h}$. All hepatic sections were examined and images were captured via light microscopy (magnification, x20 and x60; BX-50; Olympus Corporation, Tokyo, Japan). Thickenings of the perisinusoidal, periportal and pericentral spaces (26) were measured and analyzed by Olympus cellSens software version 1.12 (Olympus Corporation).

Transmission electron microscopy (TEM). Liver tissue samples, $1 \mathrm{~mm}^{3}$ in size, were taken from the livers. They were immediately fixed in $2.5 \%$ buffered glutaraldehyde for $2 \mathrm{~h}$ at room temperature. Subsequently, the specimens were immersed in $1 \%$ osmium tetroxide, dehydrated, infiltrated with propylene oxide, and embedded in pure Araldite 502 resin polymerized at room temperature for $24 \mathrm{~h}, 48^{\circ} \mathrm{C}$ for $48 \mathrm{~h}$ and $60^{\circ} \mathrm{C}$ for $48 \mathrm{~h}$. Then, thin sections $(0.5-1.0 \mu \mathrm{m})$ were 


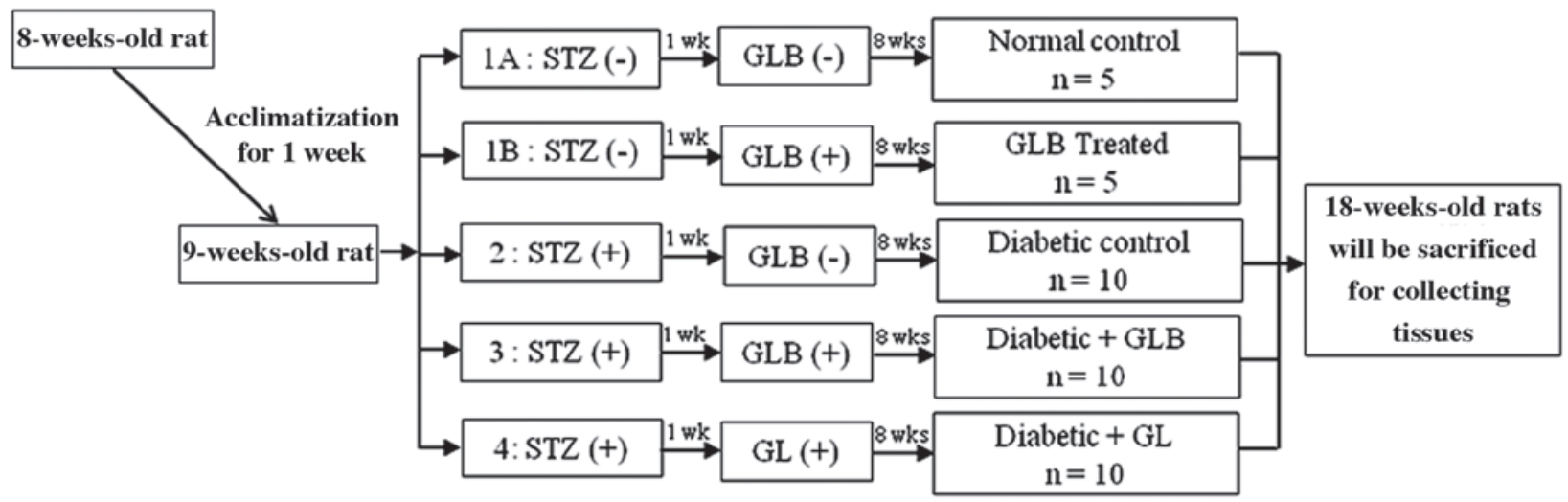

Figure 1. Experimental design. STZ, streptozotocin; GLB, glabridin; GL, glibenclamide.

stained with Toluidine blue for 2 min at room temperature, used as a guideline to identify the area of interest and further sections. Ultrathin sections $\sim 60 \mathrm{~nm}$ were processed using an ultramicrotome. Ultrathin sections were then spread mostly on 200 or 300 mesh copper grids and stained with uranyl acetate and lead citrate solutions both at room temperature for $15 \mathrm{~min}$. The section were examined and images were captured using TEM (TEM-JEM2010; JEOL, Ltd., Tokyo, Japan).

Western blot analysis of collagen type I and fibronectin. Liver lysates were prepared on ice-cold RIPA buffer (Sigma-Aldrich; Merck KGaA) supplemented with 1x protease inhibitor cocktails (EMD Millipore, Billerica, MA, USA). Homogenates were centrifuged at $14,000 \mathrm{x}$ g for $30 \mathrm{~min}$ at $4^{\circ} \mathrm{C}$ to collect supernatants. The protein concentration of the supernatant was assessed using a BCA protein assay kit (Pierce; Thermo Fisher Scientific, Inc., Waltham, MA, USA). Protein samples $(10 \mu \mathrm{g})$ of liver tissues from control, GLB, $\mathrm{DM}, \mathrm{DM}+\mathrm{GLB}$ and $\mathrm{DM}+\mathrm{GL}$ rats were diluted $1: 2$ in $2 \mathrm{X}$ treatment buffer $(0.125 \mathrm{M}$ Tris-HCl, $4 \%$ SDS, $20 \%$ glycerol, $10 \%$ 2-mercaptoethanol and $0.2 \%$ bromophenol blue) and boiled for $5 \mathrm{~min}$. Protein samples were separated by $12 \%$ polyacrylamide gel electrophoresis and then transferred $(100 \mathrm{~V}, 0.35 \mathrm{~A}$ and $300 \mathrm{~W}$ for $1.5 \mathrm{~h}$ ) onto nitrocellulose membranes (GE Healthcare, Chicago, IL, USA). The membrane was blocked with $5 \%$ non-fat dry milk in $0.1 \%$ Tween phosphate buffer (PBS-T) for $60 \mathrm{~min}$ at $4{ }^{\circ} \mathrm{C}$. Nitrocellulose blot was then probed with monoclonal antibodies for collagen type I (1:3,000; cat. no. ab34710), anti-fibronectin (1:3,000; cat. no. ab2413) and $\beta$-actin (1:5,000; cat. no. ab8227; all Abcam, Cambridge, MA, USA) for $24 \mathrm{~h}$ at $4^{\circ} \mathrm{C}$. After incubating the sample with the primary antibody, the blot was washed thrice in PBS-T, and then incubated for $2 \mathrm{~h}$ at room temperature with a goat anti-rabbit horseradish peroxidase-conjugated immunoglobulin G (1:10,000; cat. no. ab6721; Abcam) which was used as a source of secondary antibodies. Finally, the membranes were exposed to film for collagen type I and fibronectin detection by enhanced chemiluminescence (ECL) method. The ECL detection system and ECL film (GE Healthcare) were used to visualize the presence of proteins on the nitrocellulose blots. The intensity of western blot bands was quantified by densitometry, using Scion Image 4.0 software (Scion Corporation, Frederick, MD, USA).
Statistical analysis. Data are expressed as the mean \pm standard error of the mean. Statistical analysis was performed using one-way analysis of variance and Bonferroni's post hoc test. $\mathrm{P}<0.05$ was considered to indicate a statistically significant difference.

\section{Results}

Effect of glabridin on blood sugar levels and liver function test. Blood sugar levels in all animals were presented in Table I. Following 1 week, rats receiving STZ injection exhibited an increase in blood glucose levels. They were significantly elevated in DM rats $(\mathrm{P}<0.001)$ as compared with control rats until termination of the study at 8 weeks. The blood sugar levels of DM+GLB and DM+GL rats were reduced when compared with DM rats. There was a significant decrease in blood sugar levels in DM+GLB rats (5 weeks, $\mathrm{P}<0.01 ; 6-8$ weeks, $\mathrm{P}<0.001$ ) and in $\mathrm{DM}+\mathrm{GL}$ rats $(\mathrm{P}<0.001)$ at $5-8$ weeks when compared with DM rats. There were no significant differences observed in the blood sugar levels between DM+GLB and DM+GL rats for 8 weeks. The liver function test results of blood samples at 8 weeks are presented in Table II. The glutamate oxaloacetate transaminase (SGOT), glutamate pyruvate transaminase (SGPT) and alkaline phosphatase (ALP) levels in DM, $\mathrm{DM}+\mathrm{GLB}$ and $\mathrm{DM}+\mathrm{GL}$ rats were increased when compared with control rats. SGPT and ALP were significantly increased in $\mathrm{DM}$ rats $(\mathrm{P}<0.05$, and $\mathrm{P}<0.001$ respectively) when compared with control rats. Following administration with glabridin and glybenclamide, the three parameters were markedly decreased in DM+GLB and DM+GL rats when compared with DM rats; however, no significance differences were identified among $\mathrm{DM}, \mathrm{DM}+\mathrm{GLB}$ and $\mathrm{DM}+\mathrm{GR}$ rats.

Histological observations of liver tissues with Masson's trichrome staining. Hepatocytes and blood vessels of the livers in all rats throughout the 8-week experiments were radially arranged in the liver lobule (Fig. 2). A cellular plate formed of hepatocytes was directed from the periphery of the lobule to its center, which was primarily one cell thick and situated between a system of capillary sinusoids, forming a meandering and porous like structure. The spaces between these hepatic plates contained irregular dilated vessels, which were capillaries and sinusoids. The sinusoids ran in the direction of the center, 
Table I. Comparison of blood sugar levels in different groups for 8 weeks.

\begin{tabular}{lcrcrc}
\hline Week & Control $(\mathrm{mg} / \mathrm{dl})$ & GLB $(\mathrm{mg} / \mathrm{dl})$ & $\mathrm{DM}(\mathrm{mg} / \mathrm{dl})$ & $\mathrm{DM}+\mathrm{GLB}(\mathrm{mg} / \mathrm{dl})$ & $\mathrm{DM}+\mathrm{GL}(\mathrm{mg} / \mathrm{dl})$ \\
\hline 1 & $70.50 \pm 4.92$ & $67.00 \pm 5.15$ & $360.50 \pm 32.33^{\mathrm{a}}$ & $314.83 \pm 30.71^{\mathrm{a}}$ & $297.67 \pm 20.69^{\mathrm{b}}$ \\
2 & $72.00 \pm 5.49$ & $88.50 \pm 7.75$ & $290.50 \pm 34.44^{\mathrm{a}}$ & $311.67 \pm 49.73^{\mathrm{a}}$ & $241.00 \pm 36.43^{\mathrm{c}}$ \\
3 & $79.17 \pm 3.25$ & $100.50 \pm 5.68$ & $351.17 \pm 43.25^{\mathrm{a}}$ & $305.33 \pm 52.59^{\mathrm{a}}$ & $176.50 \pm 38.65^{\mathrm{c}}$ \\
4 & $73.33 \pm 3.57$ & $86.33 \pm 2.67$ & $243.67 \pm 30.75^{\mathrm{a}}$ & $170.67 \pm 41.11$ & $187.50 \pm 45.61$ \\
5 & $72.00 \pm 3.98$ & $75.67 \pm 3.55$ & $301.50 \pm 55.41^{\mathrm{a}}$ & $137.00 \pm 40.87^{\mathrm{d}}$ & $192.50 \pm 54.94^{\mathrm{c}}$ \\
6 & $73.58 \pm 3.55$ & $82.83 \pm 1.51$ & $351.00 \pm 34.82^{\mathrm{a}}$ & $69.50 \pm 13.69^{\mathrm{c}}$ & $149.00 \pm 47.48^{\mathrm{c}}$ \\
7 & $73.00 \pm 4.25$ & $83.00 \pm 4.37$ & $457.00 \pm 20.99^{\mathrm{a}}$ & $158.33 \pm 37.64^{\mathrm{c}}$ & $165.83 \pm 49.04^{\mathrm{c}}$ \\
8 & $74.67 \pm 3.31$ & $81.17 \pm 3.73$ & $353.83 \pm 35.16^{\mathrm{a}}$ & $174.50 \pm 30.99^{\mathrm{c}}$ & $160.67 \pm 52.46^{\mathrm{c}}$
\end{tabular}

Data are expressed as the mean \pm standard error of the mean. ${ }^{\mathrm{a}} \mathrm{P}<0.001,{ }^{\mathrm{b}} \mathrm{P}<0.01 \mathrm{vs}$. control; ${ }^{\mathrm{c}} \mathrm{P}<0.001,{ }^{\mathrm{d}} \mathrm{P}<0.01 \mathrm{vs}$. DM. GLB, glabridin; DM, diabetes mellitus; GL, glibenclamide.

Table II. Comparison of liver function test results in different groups at 8 weeks.

\begin{tabular}{lccccc}
\hline Parameters & Control (U/L) & GLB (U/L) & DM (U/L) & DM+GLB (U/L) & DM+GL (U/L) \\
\hline SGOT & $196.00 \pm 18.28$ & $182.17 \pm 12.28$ & $223.67 \pm 24.26$ & $212.17 \pm 29.64$ & $205.00 \pm 18.02$ \\
SGPT & $69.83 \pm 6.82$ & $63.83 \pm 7.88$ & $182.33 \pm 20.05^{\text {b }}$ & $115.67 \pm 18.56$ & $111.83 \pm 18.52$ \\
ALP & $162.33 \pm 18.27$ & $157.33 \pm 17.64$ & $837.33 \pm 55.49^{\mathrm{a}}$ & $726.50 \pm 27.57^{\mathrm{a}}$ & $766.83 \pm 87.85^{\mathrm{a}}$
\end{tabular}

Data are expressed as the mean \pm standard error of the mean. ${ }^{a} \mathrm{P}<0.001,{ }^{b} \mathrm{P}<0.05$ vs. control. GLB, glabridin; DM, diabetes mellitus; GL, glibenclamide; SGOT, glutamate oxaloacetate transaminase; SGPT, glutamate pyruvate transaminase; ALP, alkaline phosphatase.

where they drained into the central vein (Fig. 2C and F). The portal triad contained a portal venule (a branch of the portal vein), an arteriole (a branch of the hepatic artery) and bile ducts (portion of the hepatobiliary system; Fig. 2A, B, D and E). The loose connective tissue containing collagen fibers were also present and located around every blood vessel, keeping the vessels in place. Collagen was stained and indicated in blue or very light green. Hepatocyte nuclei could be observed as dark red structures within cells whereas the cytoplasm was stained red. The spaces between the formed elements of the tissue were filled with the matrix.

Liver tissues of DM rats revealed by collagen fiber deposition were increased in the portal triads (Fig. 2G and $\mathrm{H}$ ) and in the areas around the boundary of hepatocytes along the sinusoidal spaces (Fig. 2I). The blood sinusoids around the central vein were dilated (Fig. 2I). Few sinusoids were opened into the central vein. Edema appeared in the hepatocytes. The hepatocyte nuclei displayed signs of pyknosis. Hepatocytes were large polyhedral cells with large round nuclei (Fig. 2L). In addition, a number of hepatocytes contained small dense nuclei with eosinophilic cytoplasm. Another finding was that lipids accumulated in the hepatocytes and formed non-membrane bound vacuoles with peripheral nuclei. The hepatocytes radiating from the central vein exhibited a regular pattern following glabridin supplementation. The portal triad contained a portal venule, an arteriole, a bile duct and lymphatic vessels (Fig. 2I and K), which were similar in the normal portal triad of control rats. Blood sinusoids were located between plates of hepatocytes.
Administration of glabridin in DM+GLB (Fig. 2L) and glibenclamide in DM+GL (Fig. 2O) rats lowered the increase of central vein and sinusoidal dilatation, lipid accumulation, pyknotic nuclei of hepatocytes and inflammation in the area of portal triad. Decreased collagen fiber deposition in the portal triad in DM+GLB and DM+GL rats (Fig. 2I and K) were a signal of reduced fibrosis. Therefore, glabridin attenuated the severity of liver damage resulting from STZ-induced diabetes.

The area of collagen deposition in the liver tissue, stained in blue, were quantified by thickness in three zones, area of portal triad (periportal area or periportal space; PT), area around the boundary of hepatocytes along the sinusoidal space (perisinusoidal area or perisinusoidal space; PS) and around the central vein (pericentral area or pericentral space; PC) of all animal groups. The thickness of these three different zones were measured (Table III). The thickness of PT, PC and PS in DM rats $(70.71 \pm 7.13,9.92 \pm 1.74$ and $3.06 \pm 0.56 \mu \mathrm{m}$, respectively) was increased, when compared with control rats. They were significantly increased in PT and PC of DM $(\mathrm{P}<0.001)$ when compared with control rats. In contrast, thickness of PT, PC and PS was markedly reduced compared with the DM group following supplementation of DM+GLB $(38.36 \pm 6.36,5.19 \pm 0.50$ and $1.16 \pm 0.26$, respectively) and DM+GL (37.49 $\pm 3.66,5.07 \pm 0.81$ and $1.18 \pm 0.16$, respectively).

Histological changes in liver cells and tissue with TEM technique. TEM was used to observe differences in hepatic cells and tissues (Figs. 3 and 4). Micrographs of normal livers in control (Fig. 3A) and GLB (Fig. B rats exhibited round 
C

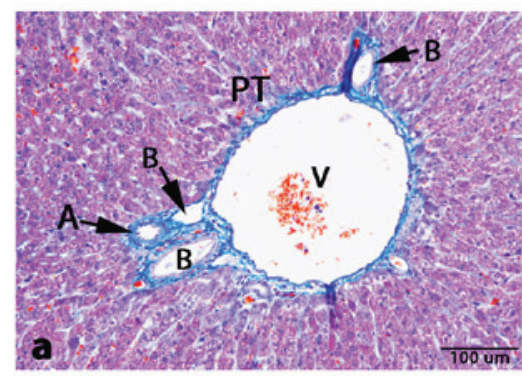

GLB

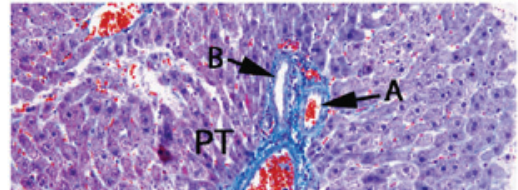

DM
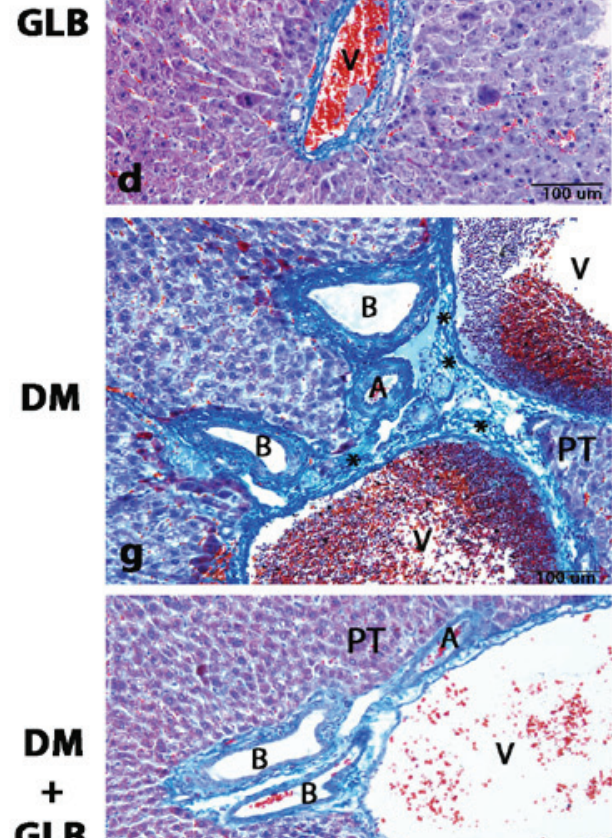

GLB
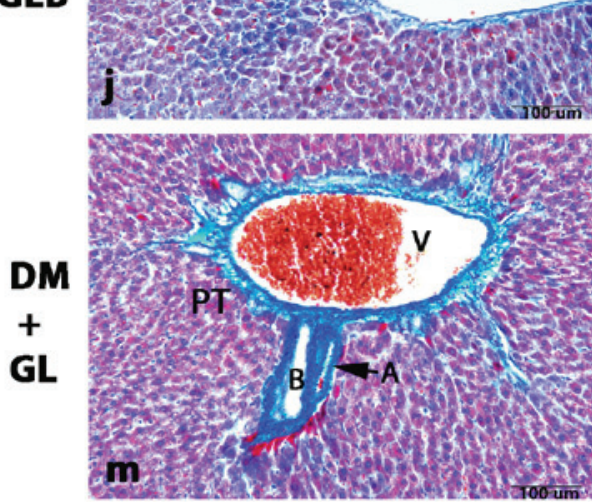

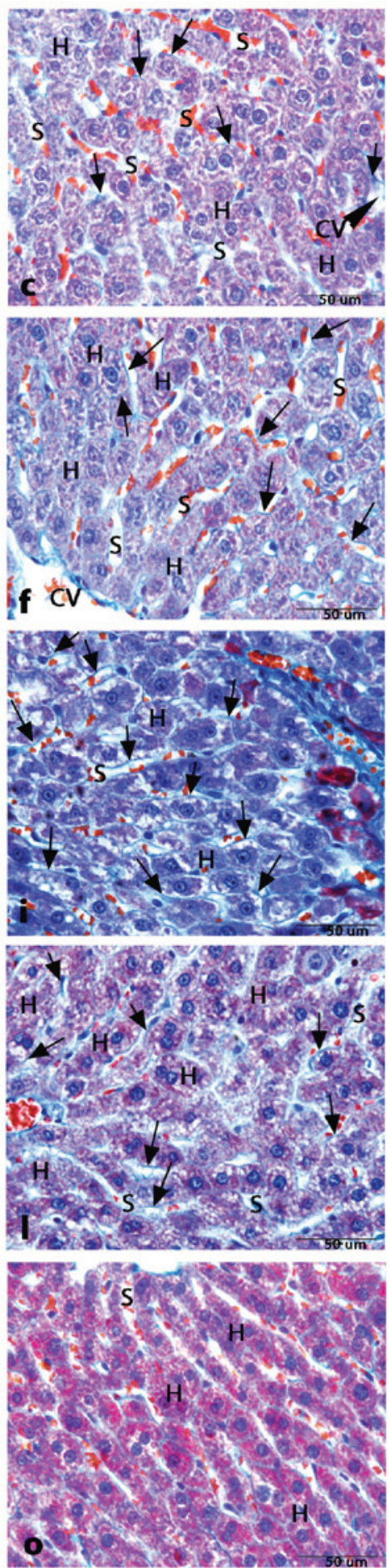

Figure 2. Photomicrographs of histological structure of liver tissue in rats by Masson's trichrome staining. (A, B, and C) Control and (D, E and F) GLB tissue exhibited a normal PT cellular architecture, consisting of V, A and B. A and D (magnification, x20). (B and E) Micrographs in the area of PT showing the normal collagen fiber deposition (magnification, $\mathrm{x} 60$ ). ( $\mathrm{C}$ and $\mathrm{F}$ ) $\mathrm{H}$ arrangement resembled that of a sponge with sinusoids presented by the $\mathrm{S}$ in the direction of the center, where they drained into the CV. Collagen fibers are stained very light blue (arrows) around the boundary of $\mathrm{H}$ (magnification, $\mathrm{x} 60$ ). (G) The DM group exhibited collagen fiber deposition which was increased at the area of PT (magnification, $\mathrm{x} 20$ ). (H) Micrograph in the area of PT illustrating the abundant of collagen fiber deposition (magnification, x60). (I) Collagen fibers deposition also increases in the area around the boundary of $\mathrm{H}$ along the sinusoids (magnification, $\mathrm{x} 60)$. The DM+GLB (J, K and L) and DM+GL groups (M, N and O) respectively illustrating the area of PT, which is similar to control and GLB rats (magnification, $\mathrm{x} 20$ ). (K and N) Micrographs in the area of PT in high magnification (magnification, x60). (L and O) Liver tissue exhibits normal collagen fiber deposition stained very light blue (arrows) around the boundary of H. (magnification, x60). *indicates collagen fiber deposition. PT, portal triad; V, portal venule; A, arteriole; B, bile duct; H, hepatocyte; CV, central vein; S, sinusoids; N, nerve bundles; DM, diabetes mellitus; GLB, glabridin; GL, glibenclamide; $\mathrm{H}$, hepatocyte; CV, central vein.

euchromatic nuclei. The cytoplasm contained numerous mitochondria, which presented with round and oval shapes inside. Smooth and rough endoplasmic reticulum were abundant whereas Golgi apparatus were dispersed in the cytoplasm. The hepatic sinusoids were lined with thin endothelial cells. The perisinusoidal space was located between hepatocytes and 
Table III. Thickness of collagen deposition in PT, PC and PS $(\mu \mathrm{m})$ in different groups.

\begin{tabular}{lccc}
\hline Group & PT $(\mu \mathrm{m})$ & PC $(\mu \mathrm{m})$ & PS $(\mu \mathrm{m})$ \\
\hline Control & $31.51 \pm 2.49$ & $4.63 \pm 0.50$ & $1.15 \pm 0.25$ \\
GLB & $31.77 \pm 1.95$ & $4.49 \pm 0.58$ & $1.13 \pm 0.18$ \\
DM & $70.71 \pm 7.13^{\mathrm{a}}$ & $9.92 \pm 1.74^{\mathrm{a}}$ & $3.06 \pm 0.56$ \\
DM+GLB & $38.36 \pm 6.36$ & $5.19 \pm 0.50$ & $1.16 \pm 0.26$ \\
DM+GL & $37.49 \pm 3.66$ & $5.07 \pm 0.81$ & $1.18 \pm 0.16$ \\
\hline
\end{tabular}

Data are expressed as the mean \pm standard error of the mean. ${ }^{\mathrm{a}} \mathrm{P}<0.001$ vs. control. PT, periportal space; PC, pericentral space; $\mathrm{PS}$, perisinusoidal space; GLB, glabridin; DM, diabetes mellitus; GL, glibenclamide.

the wall of hepatic sinusoids. Numerous microvilli extended from the surface of hepatocyte to the perisinusoidal space. (Fig. 4A) and GLB (Fig. 4B) rats. HSCs were observed in the perisinusoidal space that separated sinusoid and hepatocytes. The nuclei of the HSCs were visible with heterochromatin. Its cytoplasm was abundant and indented by fat droplets (Fig. 4A and B).

TEM examination revealed the pathological hepatocytes and liver tissues in DM rats. Hepatocytes were small and shrunken as fragmented cells. The irregular nucleus of hepatocytes exhibited damage with finger-like projections of the nuclear membrane. The degeneration and disorganization of cytoplasmic organelles was also presented (Fig. 3C). Many mitochondria of hepatocytes became swollen while huge lipid vacuoles were deposited in the cytoplasm of hepatocytes. Nuclear membranes were disrupted and nuclear chromatins were exposed. Endoplasmic reticulum cisternae and Golgi apparatus were dilated and destructed with irregular lamellar organization and large dilatations (Fig. 3D).

Blood sinusoids around the boundary of hepatocytes were also dilated (Fig. 3D). Collagen fiber deposition was increased in the area adjacent to the perisinusoidal space in DM rats (Fig. 4C and D). The variable size of collagen bundles was observed in the perisinusoidal space. After supplementation of glabridin for 8 weeks, the hepatocytes and HSCs had a similar appearance as that of the control group. Healthy hepatocytes with round nuclei indicated the regenerated hepatocytes of $\mathrm{DM}+\mathrm{GLB}$ rats and DM+GL (Fig. 3E and F, respectively). The cytoplasm contained light and dark stained swollen mitochondria and few vacuoles. TEM examination revealed the collagen production in the intracytoplasmic compartment of HSCs and these collagen fibers were radiated from cytoplasm toward the liver parenchyma. Collagen fiber deposition was decreased in the area adjacent to the perisinusoidal space in DM+GLB and $\mathrm{DM}+\mathrm{GL}$ rats.

Western blot analysis of collagen type I and fibronectin. The collagen type I and fibronectin protein bands were observed and visualized using monoclonal anti-collagen type I and anti-fibronectin antibodies. Western blot analysis established the specific collagen type I protein band at molecular weight $130 \mathrm{kDa}$ and a fibronectin protein band of molecular weight
$220 \mathrm{kDa}$ on films via ECL in control, GLB, DM, DM+GLB and DM+GL samples from rat livers (Fig. 5). These specific proteins represented the expression of type I collagen and fibronectin. The bands from DM revealed more intense protein expressions than that those of the control, GLB, DM, $\mathrm{DM}+\mathrm{GLB}$, and $\mathrm{DM}+\mathrm{GL}$ rats on ECL films. In addition, the positive control expression and characterization of $\beta$-actin protein were revealed at molecular weight $43 \mathrm{kDa}$. The $\beta$-actin protein was further used for protein analysis. The collagen type I and fibronectin expressions decreased following treatment with glabridin and glyburide when compared with DM animals. The amount of collagen type I and fibronectin proteins in DM rats were significantly increased compared with control $(\mathrm{P}<0.001)$. In DM+GLB rats, the quantity of collagen type I and fibronectin significantly increased when compared with control $(\mathrm{P}<0.001)$. However, the amount of collagen type I and fibronectin in DM+GLB were significantly decreased compared with DM rats $(\mathrm{P}<0.001$; Fig. 5).

\section{Discussion}

The diabetic model was developed by the administration of STZ to male rats. It was revealed that STZ-injected rats produced significantly increased levels of blood glucose. The increase of blood glucose levels stimulated the sorbital pathway and protein kinase $\mathrm{C}(\mathrm{PKC})$, which may lead to the production of growth factor and cytokines. Direct high glucose concentration which induces mitogen-activated protein kinase (MAPK) and PCK activation. Supplementation of glabridin revealed that the blood sugar levels were decreased. The effectiveness of glabridin from licorice extract and against the diabetogenic effects of streptozocin has also been established in rats (26).

For screening liver disease, liver function test are commonly used in clinical practice. In the present study, liver function tests were performed to observe the effects of STZ-induced diabetes on the liver at 8 weeks following STZ treatment. The levels of SGOT, SGPT and ALP were increased in the livers of DM rats when compared with control rats. Following glabridin supplementation, they were decreased when compared with DM rats. SAKP is a membrane bound enzyme glycoprotein enzyme (27). Expression of this enzyme is increased when the bile ducts become blocked. SGOT and SGPT are both enzymes that are mainly found in mitochondria of the liver (28). If liver damage is present, the enzymes are released into the bloodstream following liver cell death (29). Abnormally high concentrations of SGOT and SGPT in the blood may indicate damage to the liver (30). SGOT is normally present in liver, heart, skeletal muscle, kidneys, brain and red blood cells (31). SGOT is elevated with liver damage or heart attack. Elevated SGOT levels are not specific for liver damage, and SGOT has also been used as a cardiac marker (32). By contrast, SGPT is normally found largely in the liver. Therefore SGPT is a much more specific indicator of liver dysfunction (33).

The results from the histological observation of liver sections of DM rats indicated that sinusoids around the central veins and central veins were dilated. Edema appeared in the hepatocytes. The hepatocyte nuclei were evident of pyknosis. In addition, some hepatocytes were containing small dense nuclei and disruption of nuclear membranes. Another finding was that lipids accumulated in the hepatocytes and formed 

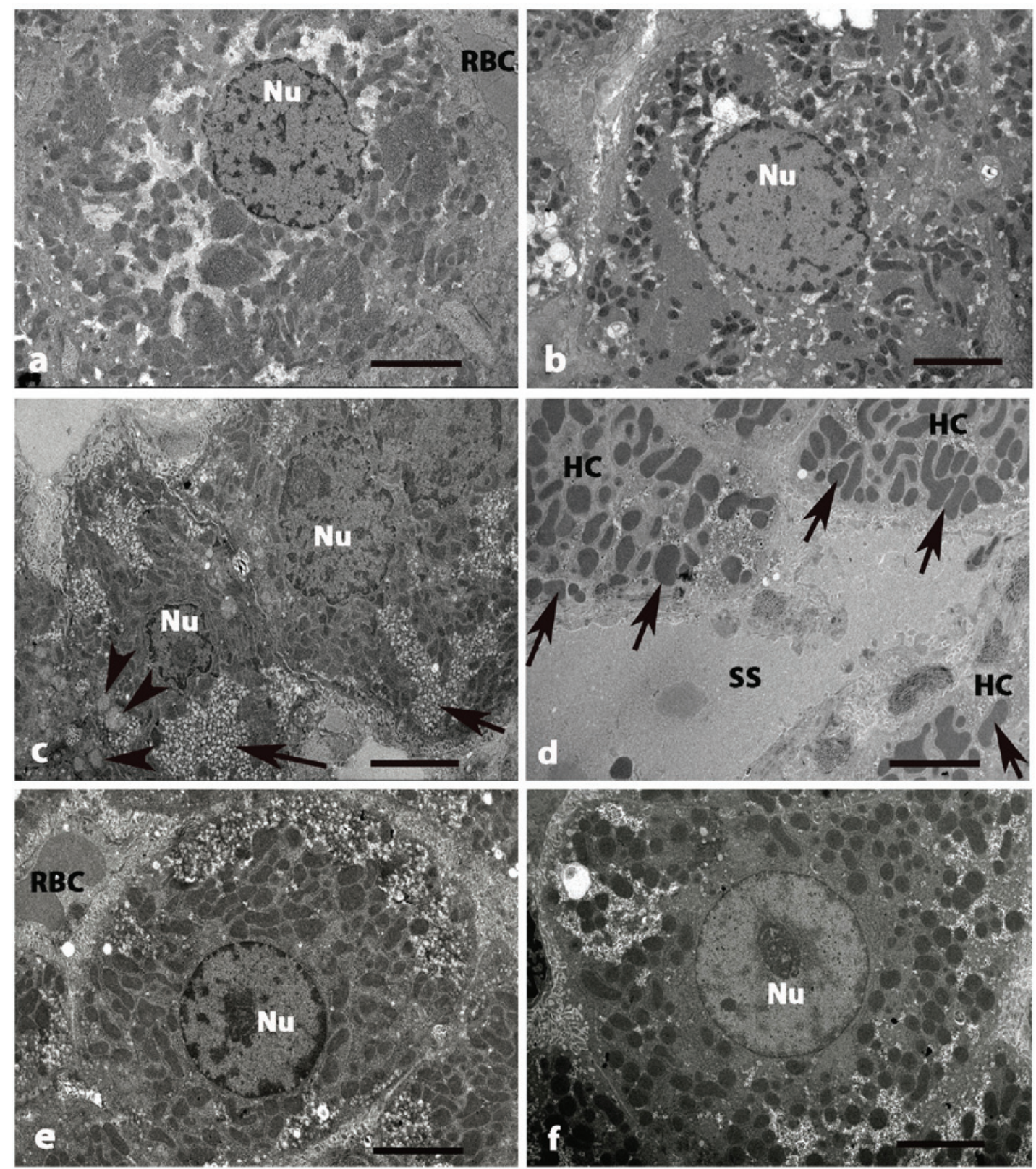

Figure 3. Electron micrographs of liver tissue in five groups of rats at 8 weeks. (A) Control and (B) GLB rats exhibited HCs with central, rounded Nu with numerous mitochondria. (C) DM rats exhibited small and fragmented HCs. The irregular Nu exhibited damage and disruption of the nuclear membranes. (D) A number of mitochondria (arrows) in HCs become swollen. Blood SS around the boundary of hepatocytes are also dilated. (E) DM+GLB and (F) DM+GL rats exhibiting the healthy hepatocyte with oval $\mathrm{Nu}$. The cytoplasm contains some lightly stained swollen mitochondria, darkly stained mitochondria and few vacuoles. Original magnification, x2,000. Scale bar, $2 \mu \mathrm{m}$. GLB, glabridin; HC, hepatocyte; Nu, nucleus; DM, diabetes mellitus; SS, sinusoids; GL, glibenclamide; RBC, red blood cell.

non-membrane bound vacuoles with peripheral nuclei. According to the results, the presented pyknosis of hepatocyte nuclei and edematous hepatocytes were displayed by continuous hepatocytes injury and death. It was evident that there were lipid droplets accumulating in the cytoplasm of hepatocytes (34). The accumulation of cytoplasmatic lipid droplets in hepatocytes is a situation that evokes the transformation into fatty liver. It is possible that this situation arises from an increased incorporation of fatty acids into the liver, which could have been a consequence of the hypoinsulinemia and the decreased capacity to excrete of lipoprotein secretion resulting from an insufficient production of apolipoprotein B (35). Characteristics of a fatty liver include the intracellular accumulation of triglycerides resulting from an increased liponeogenesis and an increased triglyceride uptake (36). Concomitantly, the hepatic secretion of very low-density lipoproteins decreases (37). The mechanism of liver damage includes cellular necrosis and inflammation, both of which are consequences of the increased mitochondrial oxidative stress from the triglyceride metabolism and the generation of free radicals in the peroxisomes (37). Another factor that increases mitochondrial oxidative stress is the increased production of adipokines (cytokines produced by the adipocytes) including tumor necrosis factor- $\alpha$ (TNF- $\alpha$ ) and leptin (38). These chemical mediators, which are produced during inflammation and cell necrosis, activate HSCs that respond by expressing 

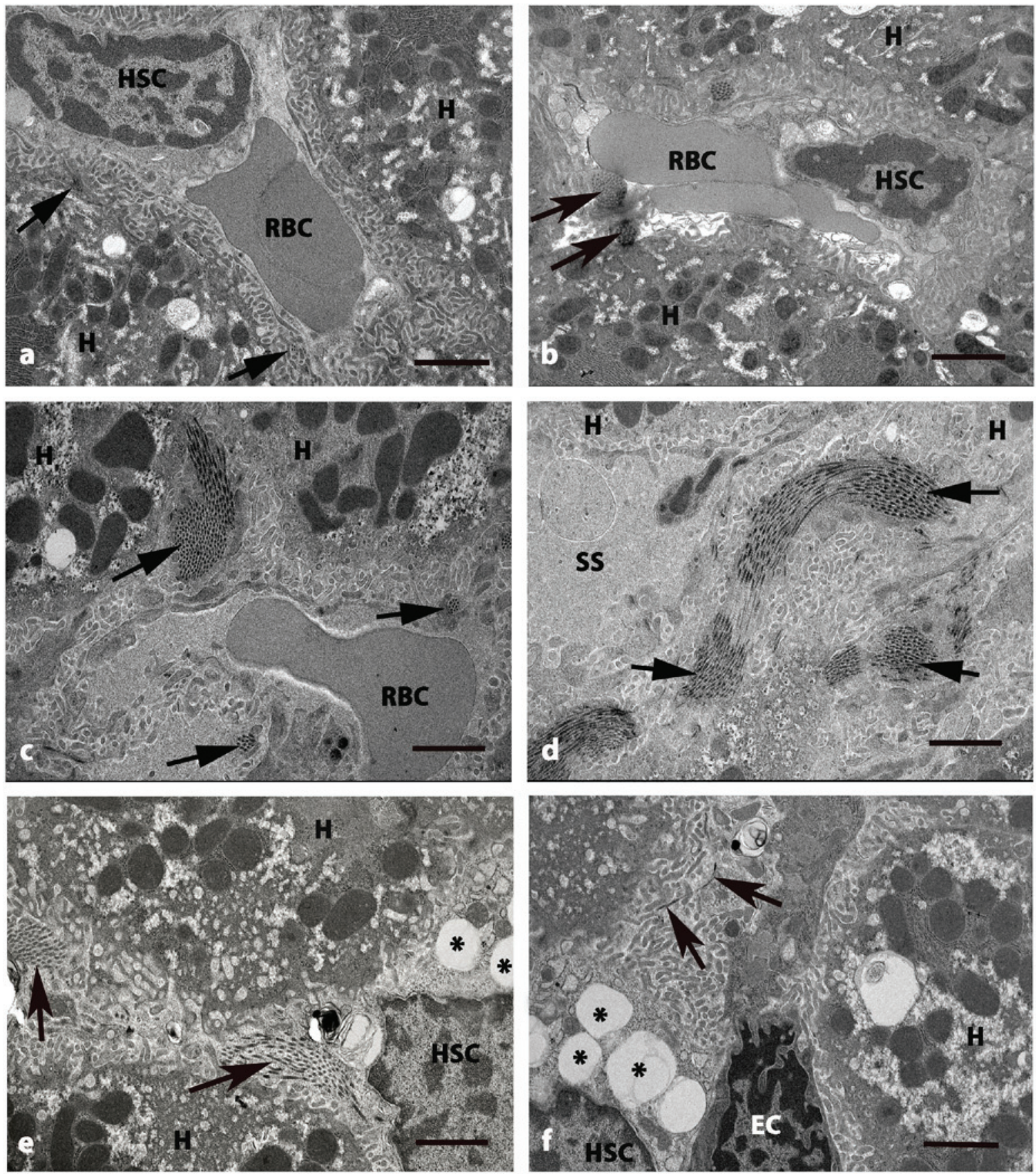

Figure 4. Electron micrographs of perisinusoidal space of liver in five groups at 8 weeks. (A) Control and (B) GLB rats exhibited H and SS that have RBCs and HSC with normal collagen bundles (arrows) at the perisinusoidal space. (C and D) DM rats exhibited swollen mitochondria in cytoplasm of $\mathrm{H}$. The deposition of collagen bundles were increased in the perisinusoidal space (arrows) and dilated blood SS. (E) DM+GLB and (F) DM+GL rats exhibited the decrease of collagen bundles in perisinusoidal space (arrows) around the boundary of H. Original magnification, x4000. Scale bar, $2 \mu \mathrm{m}$. GLB, glabridin; H, hepatocyte; SS, sinusoids; RBC, red blood cell; HSC, hepatic stellate cell; DM, diabetes mellitus; GL, glibenclamide; EC, endothelial cell.

collagen, connective tissue growth factor and aggregation of extracellular matrix components, thus leading to fibrosis $(39,40)$. Furthermore, the hepatic glycogen content of the STZ-treated animals decreased substantially. It is plausible that the displacement of glycogen in the hepatocyte cytoplasm is the result of lipid droplet accumulation (41). In addition, there are also anatomical changes of the sinusoidal capillarization. Sinusoidal endothelial cells are reported to lose fenestrae, basement membrane accumulates and sinusoidal outlets become sparse (42). These pathological alterations disturb the normal function of the sinusoid (42). Besides, capillarization of the sinusoid may disturb the exchange of several bioactive substances between the hepatocytes across, perisinusoidal space, and sinusoidal blood (43). All of these processes are to contribute to the hepatic fibrosis.

Furthermore, DM rats revealed that collagen fiber deposition was increased in the PT, PS and PC areas. Adipokines are known to activate HSCs and induce them to increase production of connective tissue growth factor, collagen, and to accumulate extracellular matrix components, in this way, favoring fibrosis (44). HSCs, also called Ito cells or perisinusoidal cells, are pericytes found in the space of Disse, 


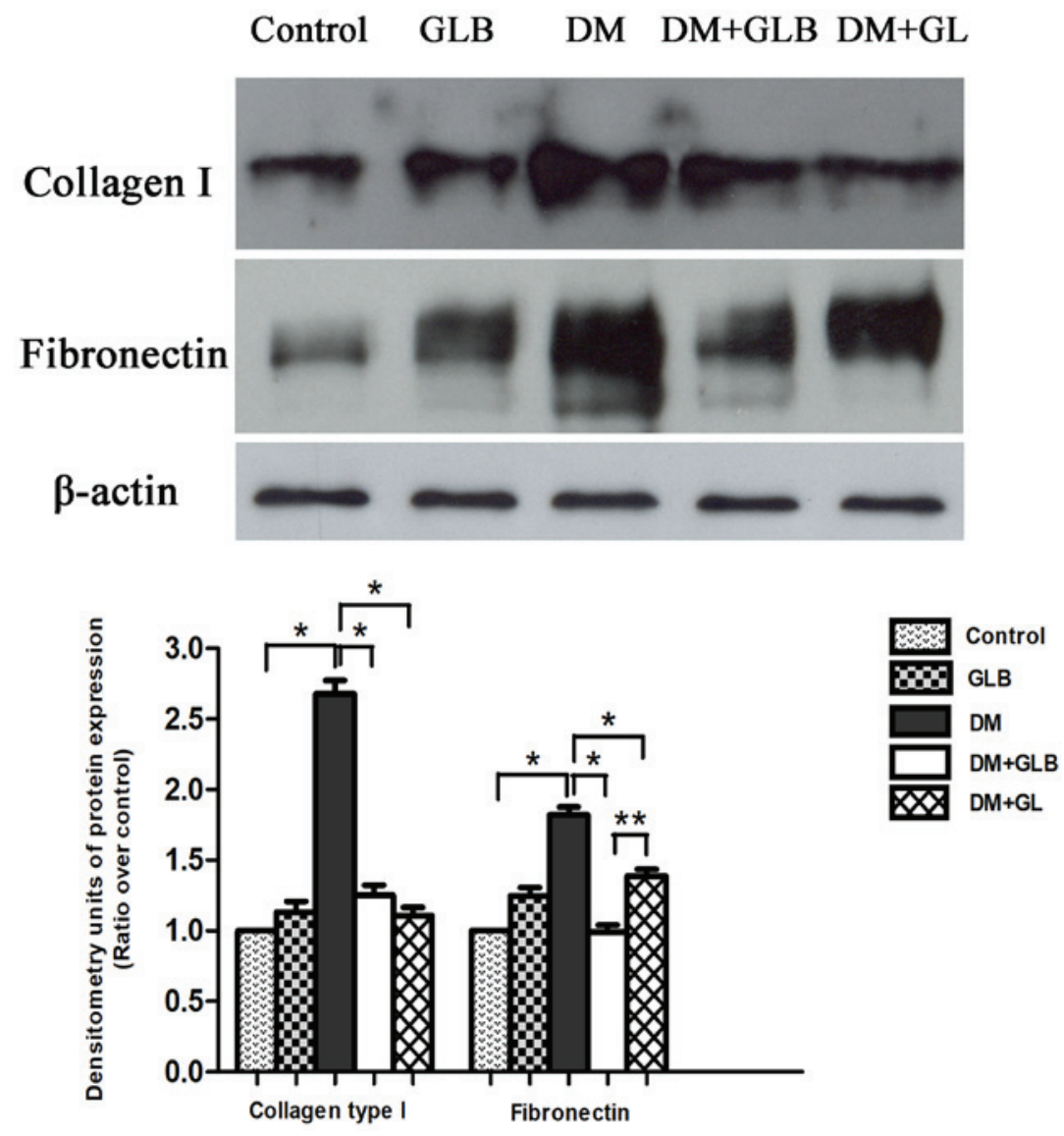

Figure 5. Western blot analysis and densitometry of protein expression demonstrated collagen type I fibronectin and $\beta$-actin proteins from liver tissues at 8 weeks. Data are expressed as the mean \pm standard error of the mean. " $\mathrm{P}<0.001$ and ${ }^{* * *} \mathrm{P}<0.01$. GLB, glabridin; DM, diabetes mellitus; GL, glibenclamide.

the hepatic space surrounding the sinusoids (45). Alteration in the microenvironment within the perisinusoidal space caused by activated HSCs facilitates the development of liver fibrosis (45). The number of activated HSCs increases in areas of inflammation (46), emphasizing the importance of resident HSCs migration into the PS during the progression of liver fibrosis. Accordingly, the number of activated HSCs is substantially reduced during the regression of liver fibrosis. This occurs by the induction of apoptosis or cellular senescence, which returns cells to a quiescent state (47). The HSCs thus constitute the main cell type participating in liver fibrosis. HSCs become activated following hepatic damage. This process is characterized by proliferation, changes in contractility, and chemotaxis. The activated HSCs secretes collagen to the scar tissue, with cirrhosis as a potential outcome (39). HSCs are also stimulated by other cells, including hepatocyte, T-lymphocyte, and Kupffer cells. The systems of stimulation are groups of cytokine and inflammatory secretions that include TNF- $\alpha$, TGF- $\beta$, insulin-like growth factor, interleukin- 6 , and interferon- $\gamma(13,14,48)$. TGF- $\beta$ constitutes an important mediator of liver fibrosis (49-51). TGF- $\beta$ causes an increase of ECM protein production that results in myofibroblast differentiation of HSCs (52). Thus, TGF- $\beta$ serves an important role in proliferation, differentiation, and morphogenesis. In hepatic fibrosis there is an increase of TGF- $\beta$ expression, which can be understood as a homeostatic response to repair damage tissue (53). Fibronectin a non-collagenous glycoprotein serving several functions, is synthesized and secreted by hepatocytes, endothelial cells, macrophages and fibroblasts (53). Fibronectin expression is associated with normal processes such as differentiation, and to pathological processes such as cellular damage, hepatic fibrosis and repair (54). The most abundant collagen types found in a healthy liver are the fibril-forming types, i.e. collagen types I and III (55). During fibrogenesis, as the collagen becomes integrated into the ECM, there is an eight-fold increase in types I and III (55). In addition, the ratio of collagen type I/III is changed from 1:1 in healthy liver to 1:2 in the cirrhotic liver (55).

Recent studies have demonstrated that the cause of the development of DM and its complications are lipid peroxidation that leads to the formation of ROS (56). An increase in ROS generation together with a decrease in the activity of antioxidant system causes an imbalance that leads to oxidative stress (57). The high level of blood sugar that occurs in diabetes results in oxidative stress and weakens the capacity of endogenous antioxidant substances due to the synthesis of several reducing sugars via both the glycolytic and polyol pathways (57).

In the present study, the demonstration of the therapeutic effect of glabridin and the drug, glibenclamide, were similar. Glibenclamide is used most commonly as a standard drug in STZ-induced diabetes (58) and used as positive control compared with glabridin from licorice. It is an effective drug to facilitate insulin release from $\beta$ cells (59). The mechanism of action of glibenclamide in hyperglycemic conditions is to lower blood glucose via stimulating insulin production from 
the existing $\beta$ cells of pancreas in STZ diabetic rats (59). In addition to this direct action, it also exhibits pancreatic effects. This drug binds to the sulfonylurea receptor 1 in the pancreatic $\beta$ cells (60). This inhibition causes cell membrane depolarization and opens the voltage dependent calcium channels (61). It is one of the leading treatments for diabetes that can increase intracellular calcium concentration in $\beta$ cells and subsequently stimulates the release of insulin (62). Glabridin can be used as a complementary and alternative therapy due to its reduced cost compared with other pharmaceutical agents and easier access to diabetes treatment (25). The hypolipidemia effect and the marked decrease in artherogenic indexes by glabridin in STZ-treated rats reduced the incidence of artherosclerosis in diabetic patients (63). Additionally, the beneficial therapeutic effect has been reviewed for the active compound from licorice; glabridin significantly elevates SOD activities which lowers MDA content of kidney, pancreas and liver. Hence, it may be concluded that part of the antioxidative activities of glabridin in STZ-induced diabetic mice may result from its hypoglycemic effects (25). Therefore, glabridin may prevent the progression of diabetic liver fibrosis, which probably acts by enhancing anti-oxidative and anti-inflammatory capacity.

In conclusion, the present findings suggest that glabridin has the potential to recover the damaged liver tissue of STZ-induced diabetic rat. The $40-\mathrm{mg} / \mathrm{kg}$ dose of glabridin from licorice results in a suitable outcome to ameliorate the pathological changes in diabetes rat hepatocytes and collagen accumulation in the extracellular matrix of the liver. Furthermore, the method of light microscopy, TEM and western blot analysis clearly elucidated the decrease of collagen deposition in the liver and was beneficial in establishing glabridin as a therapeutic target for diabetes treatment. The results of the present study suggest that further study is required to assess the mechanism and pharmacological actions of glabridin, and to assess if it may be beneficial as a therapeutic agent in the treatment of diabetes.

\section{Acknowledgements}

The authors are thankful to Mrs. Anna Chatthong for improving the English of this manuscript.

\section{Funding}

The present study was supported by a grant from Prince of Songkla University Research Fund (grant no. SCI581209S).

\section{Availability of data and materials}

The datasets used and/or analyzed during the present study are available from the corresponding author on reasonable request.

\section{Authors' contributions}

WK designed and conducted the research. JN prepared the tissue. MK and UV performed staining and western blot analysis. WK and VA wrote the manuscript and performed statistical analysis. All authors read and approved the final version of the manuscript.

\section{Ethics approval and consent to participate}

The experimental protocol used was approved by the Animal Ethics Committee of the Prince of Songkla University (Hatyai, Thailand).

\section{Patient consent for publication}

Not applicable.

\section{Competing interests}

The authors declare that they have no competing interests.

\section{References}

1. Ban CR and Twigg SM: Fibrosis in diabetes complications: Pathogenic mechanisms and circulating and urinary markers. Vasc Health Risk Manag 4: 575-596, 2008

2. Law B, Fowlkes V, Goldsmith JG, Carver W and Goldsmith EC: Diabetes-induced alterations in the extracellular matrix and their impact on myocardial function. Microsc Microanal 18: 22-34, 2012.

3. Ikeda S, Makino H, Haramoto T, Chikata K, Kumagai I and Ota Z: Changes in glomerular extracellular matrices components in diabetic nephropathy. J Diabet Complications 5: 186-188, 1991.

4. Arthur MJ: Fibrogenesis II. Metalloproteinases and their inhibitors in liver fibrosis. Am J Physiol Gastrointest Liver Physiol 279: G245-G249, 2000.

5. Das J, Roy A and Sil PC: Mechanism of the protective action of taurine in toxin and drug induced organ pathophysiology and diabetic complications: A review. Food Funct 3: 1251-1264, 2012.

6. Friedman SL: Molecular regulation of hepatic fibrosis, an integrated cellular response to tissue injury. J Biol Chem 275: 2247-2250, 2000.

7. Rockey DC: The cell and molecular biology of hepatic fibrogenesis: Clinical and therapeutic implications. Clin Liver Dis 4: 319-355, 2000.

8. Wu LL, Cox A, Roe CJ, Dziadek M, Cooper ME and Gilbert RE: Transforming growth factor betal and renal injury following subtotal nephrectomy in the rat: Role of the renin-angiotensin system. Kidney Int 51: 1553-1567, 1994.

9. Benyon RC and Iredale JP: Is liver fibrosis reversible? Gut 46 : 443-446, 2000

10. Friedman SL: Hepatic stellate cells: Protean, multifunctional, and enigmatic cells of the liver. Physiol Rev 88: 125-172, 2008.

11. Safadi R and Friedman SL: Hepatic fibrosis - role of hepatic stellate cell activation. MedGenMed 4: 27, 2002.

12. Walton KL, Johnson KE and Harrison CA: Targeting TGF- $\beta$ mediated SMAD signaling for the prevention of fibrosis. Front Pharmacol 8: 461, 2017.

13. Liu T, Wang X, Karsdal MA, Leeming DJ and Genovese F: Molecular serum markers of liver fibrosis. Biomark Insights 7: 105-117, 2012.

14. Fallatah HI: Noninvasive biomarkers of liver fibrosis: An overview. Adv Hepatol 2014: 115, 2014.

15. Manabe R, Oh-e N and Sekiguchi K: Alternatively spliced EDA segment regulates fibronectin-dependent cell cycle progression and mitogenic signal transduction. J Biol Chem 274: 5919-5924, 1999.

16. Mabuchi A, Mullaney I, Sheard P, Hessian P, Zimmermann A, Senoo $\mathrm{H}$ and Wheatley AM: Role of hepatic stellate cells in the early phase of liver regeneration in rat: Formation of tight adhesion to parenchymal cells. Comp Hepatol 1 (3 Suppl): S29, 2004

17. Ala-Kokko L, Pihlajaniemi T, Myers JC, Kivirikko KI and Savolainen ER: Gene expression of type I, III, IV collagens in hepatic fibrosis induced by dimethylnitrosamine in the rat. Biochem J 244: 75-79, 1987.

18. Yokozawa T, Cho EJ, Rhyu DY, Shibahara N and Aoyagi K: Glycyrrhizae radix attenuates peroxynitrite-induced renal oxidative damage through inhibition of protein nitration. Free Radic Res 39: 203-211, 2005.

19. Mori H, Niwa K, Zheng Q, Yamada Y, Sakata K and Yoshimi N: Cell proliferation in cancer prevention; effects of preventive agents on estrogen-related endometrial carcinogenesis model and on an in vitro model in human colorectal cells. Mutat Res 480-481: 201-207, 2001. 
20. Shang H, Cao S, Wang J, Zheng H and Putheti R: Glabridin from Chinese herb licorice inhibits fatigue in mice. Afr J Tradit Complement Altern Med 7: 17-23, 2009.

21. Yokota T, Nishio H, Kubota Y and Mizoguchi M: The inhibitory effect of glabridin from licorice extracts on melanogenesis and inflammation. Pigment Cell Res 11: 355-361, 1998.

22. Choi EM: The licorice root derived isoflavan glabridin increases the function of osteoblastic MC3T3-E1 cells. Biochem Pharmacol 70: 363-368, 2005.

23. Gnanamoorthy M, Sridharan K, Dhayananth N and Ramesh Babu NG: Antimicrobial and anticancer potential of glycyrrhiza glabra. IJERM 4: 2349-2058, 2017.

24. Jung JC, Lee YH, Kim SH, Kim KJ, Kim KM, Oh S and Jung YS Hepatoprotective effect of licorice, the root of Glycyrrhiza uralensis Fischer, in alcohol induced fatty liver disease. BMC Complement Altern Med 16: 19, 2016.

25. Wu F, Jin Z and Jin J: Hypoglycemic effects of glabridin, a polyphenolic flavonoid from licorice, in an animal model of diabetes mellitus. Mol Med Rep 7: 1278-1282, 2013.

26. Hui AY, Liew CT, Go MY, Chim AM, Chan HL, Leung NV and Sung JJ: Quantitative assessment of fibrosis in liver biopsies from patients with chronic hepatitis B. Liver Int 24: 611-618, 2004.

27. Stinson RA and Hamilton BA: Human liver plasma membranes contain an enzyme activity that removes membrane anchor from alkaline phosphatase and converts it to a plasma-like form. Clin Biochem 27: 49-55, 1994.

28. Wilson PD, Franks LM, Cottell DC and Benham F: Alkaline phosphatase in mitochondria. Cell Biol Int Rep 1: 85-92, 1977.

29. Jeschke MG: The hepatic response to thermal injury: Is the liver important for postburn outcomes? Mol Med 15: 337-351, 2009.

30. Tian Z, Liu H, Su X, Fang Z, Dong Z, Yu C and Luo K: Role of elevated liver transaminase levels in the diagnosis of liver injury after blunt abdominal trauma. Exp Ther Med 4: 255-260, 2012.

31. Giannini EG, Testa R and Savarino V: Liver enzyme alteration: A guide for clinicians. CMAJ 172: 367-379, 2005.

32. Shen J, Zhang J, Wen J, Ming Q, Zhang J and Xu Y: Correlation of serum alanine aminotransferase and aspartate aminotransferase with coronary heart disease. Int J Clin Exp Med 8: 4399-4404, 2015.

33. Schneeberger EE, Arriola MS, Fainboim H, Schroder T, González J, Baiges D, Luque M, Maldonado Coco JA and Citera G: Idiophatic inflammatory myophaties: Its association with liver disorders. Rev Fac Cien Med Univ Nac Cordoba 69: 139-143, 2012 (In Spanish).

34. Hosseinzadeh $\mathrm{H}$ and Nassiri-Asl M: Pharmacological effects of glycyrrhiza spp. And its bioactive constituents: Update and review. Phytother Res 29: 1868-1886, 2015.

35. Bilal HM, Riaz F, Munir K, Saqib K and Sawa MR: Histological changes in the liver of diabetic rats: A review of pathogenesis of nonalcoholic fatty liver disease in type 1 diabetes mellitus. Cogent Medicine 3: 1275415, 2016.

36. Kawano Y and Cohen DE: Mechanisms of hepatic triglyceride accumulation in non-alcoholic fatty liver disease J Gastroenterol 48: 434-441, 2013.

37. Nakashima O, Kurogi M, Yamaguchi R, Miyaaki H, Fujimoto M, Yano H, Kumabe T, Hayabuchi N, Hisatomi J, Sata M and Kojiro M: Unique hypervascular nodules in alcoholic liver cirrhosis: Identical to focal nodular hyperplasia-like nodules. J Hepatol 41: 992-998, 2004.

38. Pessayre D, Fromenty B and Mansouri A: Mitochondrial injury in steatohepatitis. Eur J Gastroenterol Hepatol 16: 1095-1105, 2004.

39. Crespo J, Cayon A, Fernandez-Gil P, Hernandez-Guerra M, Mayorga M, Dominguez-Diez A, Fernandez-Escalante JC and Pons-Romero F: Gene expression of tumor necrosis factor alpha and TNF-receptors, p55 and p75, in nonalcoholic steatohepatitis patients. Hepatology 34: 1158-1163, 2001.

40. Sanyal AJ; American Gastroenterological Association: AGA technical review on nonalcoholic fatty liver disease. Gastroenterology 123: 1705-1725, 2002.

41. Bertolani $\mathrm{C}$ and Marra F: The role of adipokines in liver fibrosis Pathophysiology 15: 91-101, 2008.

42. Poisson J, Lemoinne S, Boulanger C, Durand F, Moreau R, Valla D and Rautou PE: Liver sinusoidal endothelial cells: Physiology and role in liver diseases. J Hepatol 66: 212-227, 2017.

43. Ohno T, Horio F, Tanaka S, Terada M, Namikawa T and Kitch J: Fatty liver and hyperlipidemia in IDDM (insulin dependent diabetes mellitus) of streptozotocin treated shrew. Life Sci 66: 125-131, 2000.
44. Onori P, Morini S, Franchitto A, Sferra R, Alvaro D and Gaudio E: Hepatic microvascular features in experimental cirrhosis: A structural and morphometrical study in CCI4-treated rats. J Hepatol 33: 555-563, 2000

45. Hynes RO: The extracellular matrix; not just pretty fibrils Science 326: 1216-1219, 2010.

46. Yerian L: Identifying activated hepatic stellate cells in chronic and posttransplant recurrent hepatitis C. Liver Transpl 14 756-758, 2008

47. Friedman SL: The virtuosity of hepatic stellate cells. Gastroenterology 117: 1244-1246, 1999.

48. Inagaki Y and Okazaki I: Emerging insights into Transforming growth factor beta Smad signal in hepatic fibrogenesis. Gut 56: 284-292, 2007.

49. Meindl-Beinker NM and Dooley S: Transforming growth factor-beta and hepatocyte transdifferentiation in liver fibrogenesis. J Gastroenterol Hepatol 23 (Suppl 1): S122-S127, 2008.

50. Arauz J, Zarco N, Segovia J, Shibayama M, Tsutsumi V and Muriel P: Caffeine prevents experimental liver fibrosis by blocking the expression of TGF- $\beta$. Eur J Gastroenterol Hepatol 26: 164-273, 2014.

51. Gressner AM and Weiskirchen R: Modern pathogenetic concepts of liver fibrosis suggest stellate cells and TGF-beta as major players and therapeutic targets. J Cell Mol Med 10: 76-99, 2006.

52. Mosesson MW: Fibrinogen and fibrin and structure and functions. J Thromb Haemost 3: 1894-1904, 2005.

53. Gressner OA, Weiskirchen R and Gressner AM: Biomarkers of liver fibrosis: Clinical translation of molecular pathogenesis or based on liver dependent malfunction tests. Clin Chim Acta 381: 107-113, 2007.

54. Kawelke N, Vasel M, Sens C, Au AV,Dooley S and Nakchbandi IA: Fibronectin protects from excessive liver fibrosis by modulating the availability of and responsiveness of stellate cells to active TGF- $\beta$. PLoS One 6: e28181, 2011

55. Liu XY, Liu RX, Hou F, Cui LJ, Li CY, Chi C, Yi E, Wen Y and Yin CH: Fibronectin expression is critical for liver fibrogenesis in vivo and in vitro. Mol Med Rep 14: 3669-3675, 2016.

56. Masarone M, Rosato V, Dallio M, Gravina AG, Aglitti A, Loguercio C, Federico A and Persico M: Role of oxidative stress in pathophysiology of nonalcoholic fatty liver disease. Oxid Med Cell Longev 2018: 9547613, 2018.

57. Meng XM, Chung AC and Lan HY: Role of the TGF- $\beta /$ BMP-7/ Smad pathways in renal diseases. Clin Sci (Lond) 124: 243-254, 2013.

58. Gandhi GR and Sasikumar P: Antidiabetic effect of Merremia emarginata Burm. F. in streptozotocin induced diabetic rats. Asian Pac J Trop Biomed 2: 281-286, 2012.

59. Patanè G, Piro S, Anello M, Rabuazzo AM, Vigneri R and Purrello F: Exposure to glibenclamide increases rat beta cells sensitivity to glucose. Br J Pharmacol 129: 887-892, 2000.

60. Oh CS, Kohanim S, Kong FL, Song HC, Huynh N, Mendez R, Chanda M, Edmund Kim E and Yang DJ: Sulfonylurea receptor as a target for molecular imaging of pancreas beta cells with (99m)Tc-DTPA-glipizide. Ann Nucl Med 26: 253-261, 2012.

61. Petit P and Loubatières-Mariani MM: Potassium channels of the insulin-secreting B cell. Fundam Clin Pharmacol 6: 123-134, 1992.

62. Serrano-Martín X,Payares G and Mendoza-León A: Glibenclamide, a blocker of $\mathrm{K}+(\mathrm{ATP})$ channels, shows antileishmanial activity in experimental murine cutaneous leishmaniasis. Antimicrob Agents Chemother 50: 4214-4216, 2006.

63. Abd EI-Ghffar EA: Ameliorative effect of glabridin, a main component of Glycyrrhiza glaba L. roots in streptozotocin induced Type I diabetes in male albino rats. Indian $\mathbf{J}$ Tradit Knowle 15: 570-579, 2016.

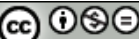

This work is licensed under a Creative Commons Attribution-NonCommercial-NoDerivatives 4.0 International (CC BY-NC-ND 4.0) License. 